\title{
Beware of Drug Interactions Treating latrogenic Mycobacterium bovis Endocarditis from Intravesical BCG Instillation
}

\author{
Francesca Curri ${ }^{1}$, Andrea Da Porto ${ }^{1}$, Viviana Casarsa ${ }^{1}$, Daria Albini ${ }^{1}$, Giorgio Minen ${ }^{2}$, Leonardo A Sechi ${ }^{1}$ \\ ${ }^{1}$ Department of Experimental and Clinical Medical Sciences, Clinica Medica, University Hospital, University of Udine, Udine, Italy \\ ${ }^{2}$ Department of Cardiac Sciences, Cardiology, University Hospital, University of Udine, Udine, Italy
}

Received: 02/01/2021

Accepted: 08/01/2021

Published: 01/02/2021

\begin{abstract}
How to cite this article: Curri F, Da Porto A, Casarsa V, Albini D, Minen G, Sechi LA. Beware of drug interactions treating iatrogenic Mycobacterium bovis endocarditis from intravesical BCG instillation. EJCRIM 2021;8: doi:10.12890/2021_002263.
\end{abstract}

Conflicts of Interests: The Authors declare that there are no competing interests.

This article is licensed under a Commons Attribution Non-Commercial 4.0 License

\section{ABSTRACT}

We report a case of mechanical mitral valve endocarditis associated with miliary disseminated bacillus Calmette-Guerin (BCG) infection following intravesical instillations for minimally invasive bladder cancer in a 65-year-old man. The diagnosis was established by echocardiographic evidence of vegetation on the prosthetic mitral valve, miliary lesions in the lungs and evidence of bloodstream infection sustained by Mycobacterium. We successfully treated the patient with the classical regimen of quadruple antituberculous therapy.

\section{LEARNING POINTS}

- To consider endocarditis as an extra-pulmonary manifestation of Mycobacterium bovis infection, especially in the presence of predisposing heart conditions and risk factors.

- To be careful of drug interactions among warfarin, rifampin and amiodarone.

\section{KEYWORDS}

BCG, infective endocarditis, disseminated mycobacterial disease

\section{CASE DESCRIPTION}

We present a case of a 65-year-old Caucasian man who was admitted to the Emergency Department in May 2020 for the appearance of diffuse arthromyalgia and fever up to $39.2^{\circ} \mathrm{C}\left[102.56^{\circ} \mathrm{F}\right]$ the day after intravesical instillation of BCG. On presentation, the patient was febrile, he had tachycardia (HR 120 bpm), tachypnoea (respiratory rate 22/minute) and mild hypotension (BP 100/70 mmHg). He denied other relevant symptoms. The physical examination revealed heart murmur on the heart apex and scattered crackles on chest auscultation. The ECG showed AF rhythm with complete left bundle block. The chest x-ray showed multiple peripheral areas of dense air-space opacity and hazy shadow on the lung fields with pleural effusion (Fig. 1). Bedside point-of-care ultrasonography showed reduced collapsibility of the inferior vena cava, mild bilateral pleural effusion, diffuse and asymmetric B-lines on lung examination and suspect vegetation on the mitral valve. The most relevant blood tests are reported in Table 1. A nasopharyngeal swab for SARS-CoV-2 was negative.

The patient had a history of minimally invasive bladder cancer (T1NOM0) treated with transurethral resection followed by multiple (16) intravesical instillations of BCG from December 2019 to May 2020. His medical history was notable for post-rheumatic valvular heart disease with replacement of the aortic and mitral valves with mechanical prostheses in 2005, chronic heart failure (NYHA class II), permanent atrial fibrillation, complete left bundle branch block, previous transient ischaemic attack in 2012 and stage 1 chronic obstructive pulmonary disease. 

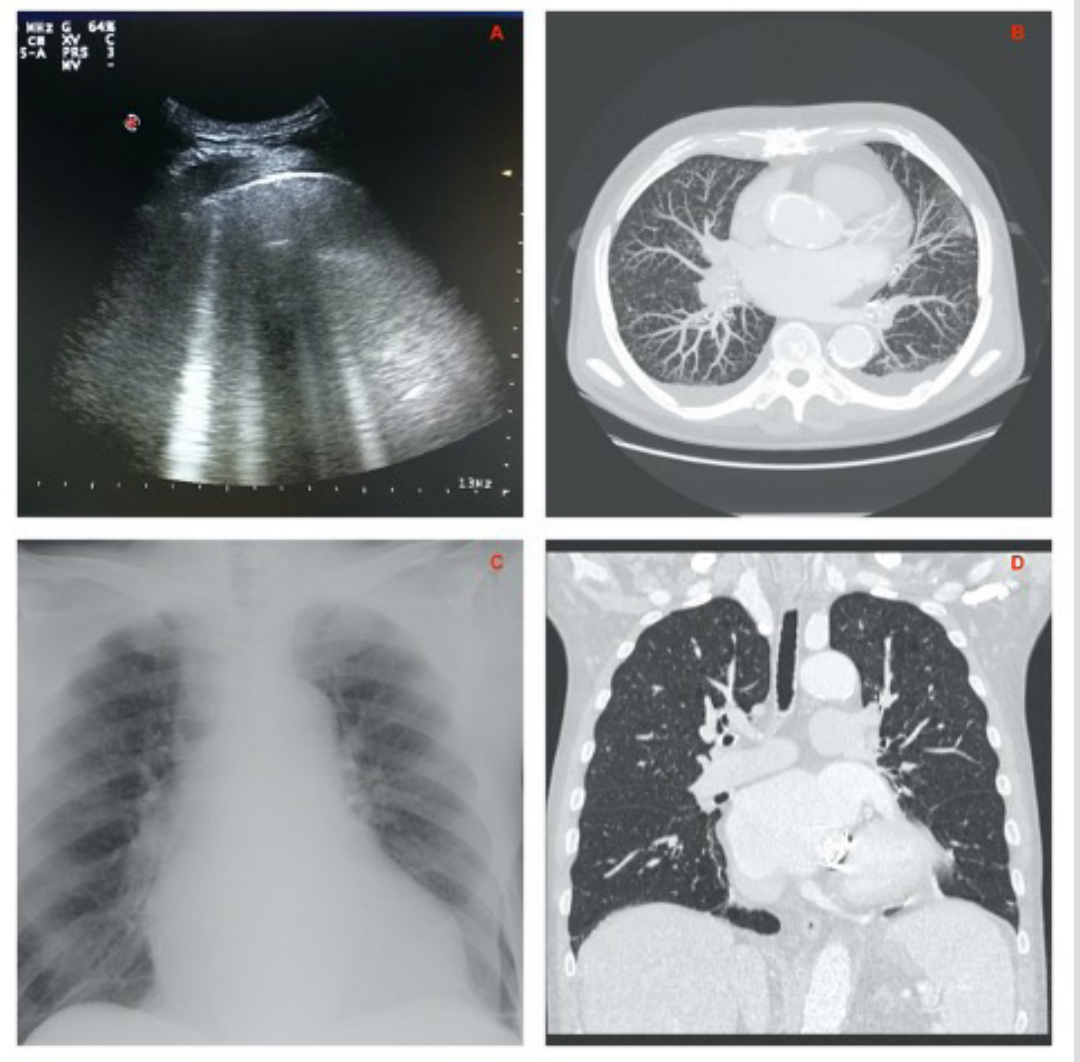

Figure 1. A) Lung ultrasound, B) lung parenchyma $3 D C T$ reconstruction, $C$ ) chest $x$-ray, $D$ ) chest $C$ T lung window (coronal) (Udine Hospital, July 2020)

\begin{tabular}{|c|c|c|}
\hline Laboratory Test & Value & Laboratory Reference Ranges \\
\hline White blood cells & $4.00 \times 10^{3} / \mu l$ & $4.00-11.00$ \\
\hline Red blood cells & $3.99 \times 10^{6} / \mu l$ & $4.60-5.60$ \\
\hline Haemoglobin & $12.3 \mathrm{~g} / \mathrm{dl}$ & $14.0-18.0$ \\
\hline Platelets & $95,000 \times 10^{3} / \mu l$ & $150-400$ \\
\hline Creatinine & $1.25 \mathrm{mg} / \mathrm{dl}$ & $0.67-1.17$ \\
\hline C-reactive protein $(C R P)$ & $91.57 \mathrm{mg} / \mathrm{l}$ & $0.00-5.00$ \\
\hline Procalcitonin (PCT) & $5.71 \mathrm{ng} / \mathrm{ml}$ & $<0.10$ \\
\hline Brain natriuretic peptide (BNP) & $4,066 \mathrm{pg} / \mathrm{ml}$ & $2-100$ \\
\hline High-sensitivity cardiac troponin (hs-Tnl) & $202.9 \mathrm{ng} / \mathrm{l}$ & $<78.5$ \\
\hline Arterial blood gas (ABG) & $\begin{array}{c}\text { pH 7.52, pCO2 } 28 \mathrm{mmHg}, \mathrm{pO} 265 \mathrm{mmHg} \\
\text { FiO2 } 21 \%, \mathrm{HCO} 25.6 \mathrm{mmol} / \mathrm{l} \\
\text { Lac } 1.4 \mathrm{mmol} / \mathrm{l}\end{array}$ & \\
\hline
\end{tabular}

Table 1. The most relevant blood test results on presentation

His home therapy consisted of warfarin $5 \mathrm{mg}$ according to INR (usually $3.75 \mathrm{mg}$ ), amiodarone $200 \mathrm{mg}$, furosemide $25 \mathrm{mg}$, pantoprazole 20 $\mathrm{mg}$, metoprolol $50 \mathrm{mg}$ and delorazepam 20 drops per day.

Based on clinical presentation, laboratory and instrumental findings at the bedside we made the diagnosis of sepsis with concurrent worsening of heart failure. Endocarditis was suspected with the patient having fever, a predisposing heart condition, heart murmur and evidence of mitral vegetation not previously known from bedside cardiac ultrasound. We focused on the most plausible aetiologies of a valvular vegetation: infection sustained by common pathogens or thrombus. 
Before starting empirical broad-spectrum antibacterial therapy (daptomycin and piperacillin-tazobactam plus amikacin) multiple bacteriological specimens were collected for culture. Blood culture (8 sets) and urine cultures were always negative for common pathogens. The patient was started on antituberculous treatment (isoniazid, rifampin, pyrazinamide and ethambutol combination) combined with methylprednisolone $60 \mathrm{mg}$ for 5 days. We reported some complexities during the 8 weeks of hospitalization: first, the mitral valve vegetation could not be removed with cardiac open surgery or minimally invasive thoracic surgery due to the disadvantageous risk/benefit ratio (ASA 4). Second, we reported 2 close episodes of symptomatic rapid ventricular response atrial fibrillation with subsequent pulmonary oedema. Therapeutic drug monitoring revealed an amiodarone plasma concentration below the therapeutic range. No recurrence of symptoms was observed after dose adjustments of amiodarone based on serum concentrations. Third, it was difficult to manage anticoagulant therapy and eventually we needed to almost quadruple the usual dosage of warfarin (from 3.75 to $12.5 \mathrm{mg} /$ day) to maintain the target INR range ( 2.5 to 3.5).

The patient was eventually discharged in early July 2020 in good clinical condition, with an echocardiographic follow-up schedule and with a 4-month combination of isoniazid and rifampin therapy.

Methods and Procedures

Transthoracic and transoesophageal echocardiograms confirmed the diagnosis of endocarditis, showing a mitral valve vegetation (16 $\mathrm{mm} \times$ $7 \mathrm{~mm}$ or $0.63 \mathrm{in} \times 0.27 \mathrm{in}$ ) partially attached to the ventricular side of the posterior cusp with no perivalvular regurgitation (Fig. 2). Chest CT and lung parenchyma 3D CT reconstruction revealed diffuse miliary pulmonary microgranules (Fig. 1), bilateral pleural effusion and several small mediastinal and aortocaval lymph nodes.
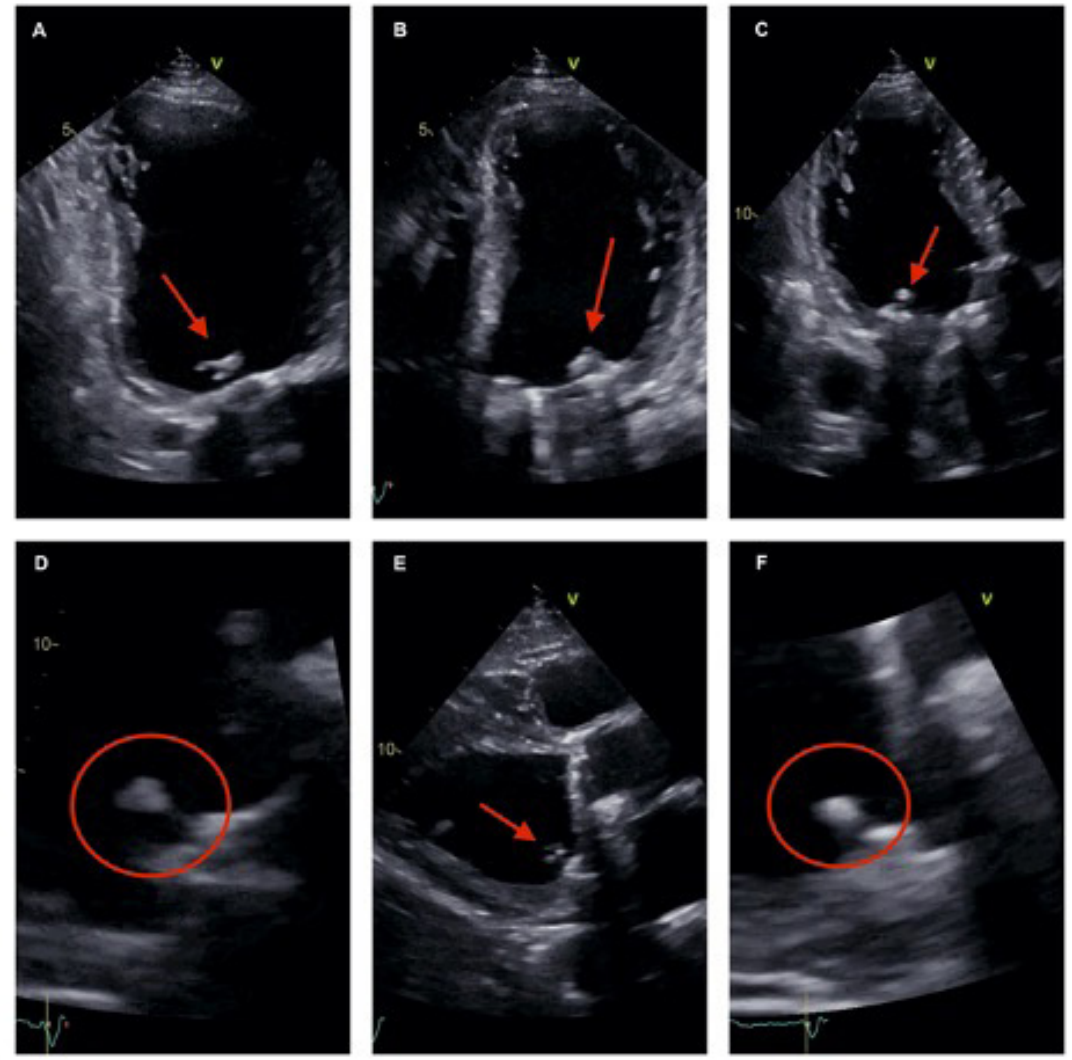

Figure 2. Transthoracic echocardiography (Department of Cardiac Sciences, Cardiology, Udine Hospital, July 2020)

Based on the CT findings and the temporal connection with the BCG bladder instillations, we suspected haematogenous spread of BCG eventually confirmed by positive polymerase chain reaction testing for the Mycobacterium tuberculosis complex in blood samples. Upon further analysis, the patient did not have a history of tuberculosis; the interferon-gamma release assay (IGRA) and Mantoux test were negative; sputum culture was also negative for mycobacterial species. The patient was HIV-negative. Three weeks later we received microbiological confirmation that the blood culture was positive for Mycobacterium bovis sp. 
No complications were reported at the 30-day follow-up and at the 3-month follow-up.

In conclusion, this case is interesting because of both the atypical clinical presentation and its management complexity. Certainly, a biopsy would have been interesting for confirmatory histological diagnosis of Mycobacterium bovis endocarditis in a disseminated mycobacterial disease scenario.

\section{DISCUSSION}

Complications of BCG infection have been reported with an incidence of 10-15\%. The complications can be mild, such as irritative bladder symptoms, weakness and fever or, in rare instances, severe, causing disseminated disease with miliary pneumonitis, granulomatous hepatitis, soft tissue infections, bone marrow involvement and life-threatening sepsis ${ }^{[1-3]}$. Very few cases of Mycobacterium bovis BCG endocarditis have been previously reported in the scientific literature. To our knowledge, ours is the third case reported. The first case was 1 of an 85-year-old man with a history of bladder cancer treated with transurethral resection and intravesical instillations of $\mathrm{BCG}^{[4]}$ who was hospitalized complaining of fever, asthenia and weight loss. A transoesophageal echocardiogram showed a vegetation on the bicuspid aortic valve, suggesting infectious endocarditis. Acid-fast bacilli were found in blood, sputum and stool samples. Cultures of these 3 types of sample were negative for common bacteria but were positive for BCG. The patient's general condition soon became unstable and he died 2 weeks later. The second case was 1 of an 86-year-old male who developed aortic blood culture-negative endocarditis (BCNE) following intravesical BCG instillations for bladder cancer but specific blood cultures for mycobacteria in Middlebrook liquid medium then identified Mycobacterium bovis ${ }^{[5]}$. Moreover, we cite the retrospective study of Pérez-Jacoiste Asín et al ${ }^{[8]}$, which included 256 patients treated with intravesical BCG during a 6-year period. They reported disseminated infection as the most common manifestation in 97 cases. Specifically, complications were miliary tuberculosis in 71 cases, fever in 19 and sepsis in 7, as occurred in our case. Pérez-Jacoiste Asín et al. ${ }^{[8]}$ had 19 cases of vascular complications due to haematogenous spread of BCG. They referred to forms of mycotic aneurysm or infection of prosthetic vascular grafts while we reported infection of a prosthetic heart valve. In fact, our case seems to be the first report of Mycobacterium bovis BCG endocarditis in a mechanical valve in a disseminated mycobacterial disease scenario. Our patient responded to therapy and no complications have been reported during follow-up. We started antituberculous therapy early, on the basis of high clinical suspicion and a positive TB-PCR test for the blood sample, without waiting for specific cultural confirmation of tuberculosis that could have delayed treatment, and perhaps worsened the outcome. An additional noteworthy aspect to mention concerns the drug interaction we reported among warfarin, rifampin and amiodarone. As is well known in the literature ${ }^{[6]}$, the concomitant administration of warfarin and rifampin resulted in the need for an unusually high maintenance dose of warfarin in order to obtain a therapeutic effect. Likewise, the drugdrug interaction between amiodarone and rifampin is well known. The long half-life of amiodarone and its active metabolite in combination with the late onset and offset of cytochrome P4503A (CYP3A4) induction by rifampin makes this a challenging drug-drug interaction to address in clinical practice ${ }^{[7]}$. Its complexity, both clinical and pharmacokinetic, can be managed by dose adjustments of amiodarone based on serum concentrations.

\section{REFERENCES}

1. Gonzalez OY, Musher DM, Brar I, Furgeson S, Boktour MR, Septimus EJ, et al. Spectrum of bacille Calmette-Guérin (BCG) infection after intravesical BCG immunotherapy. Clin Infect Dis 2003;36(2):140-148.

2. Nadasy KA, Patel RS, Emmett M, Murillo RA, Tribble MA, Black RD, et al. Four cases of disseminated Mycobacterium bovis infection following intravesical BCG instillation for treatment of bladder carcinoma. South Med J 2008;101(1):91-95.

3. Lamm DL, van der Meijden PM, Morales A, Brosman SA, Catalona WJ, Herr HW, et al. Incidence and treatment of complications of bacillus Calmette-Guerin intravesical therapy in superficial bladder cancer. J Urol 1992;147(3):596-600.

4. Fournier A, Gouriet F, Fournier PE, Casalta JP, Saby L, Habib G, et al. A case of infectious endocarditis due to BCG. Int J Infect Dis 2015;35:27-28.

5. Fournier PE, Gouriet F, Casalta JP, Lepidi H, Chaudet H, Thuny F, et al. Blood culture-negative endocarditis: improving the diagnostic yield using new diagnostic tools. Medicine (Baltimore) 2017;96(47):e8392.

6. US Food and Drug Administration [Internet]. Clinical Drug Interaction Studies - Cytochrome P450 Enzyme- and Transporter-Mediated Drug Interactions Guidance for Industry [2020]. Available from: https://www.fda.gov/regulatory-information/search-fda-guidance-documents/clinical-drug-interaction-studies-cytochrome-p450-enzymeand-transporter-mediated-drug-interactions.

7. Rotmensch HH, Belhassen B, Swanson BN, Shoshani D, Spielman SR, Greenspon AJ, et al. Steady-state serum amiodarone concentrations: relationships with antiarrhythmic efficacy and toxicity. Ann Intern Med 1984;101(4):462-469.

8. Pérez-Jacoiste Asín MA, Fernández-Ruiz M, López-Medrano F, Lumbreras C, Tejido A, San Juan R, et al. Bacillus Calmette-Guérin (BCG) infection following intravesical BCG administration as adjunctive therapy for bladder cancer: incidence, risk factors, and outcome in a single-institution series and review of the literature. Medicine (Baltimore) 2014;93(17):236-254. 